\title{
Article \\ Analytical Study of a $\phi-$ Fractional Order Quadratic Functional Integral Equation
}

\author{
Ahmed M. A. El-Sayed ${ }^{1, *,+} \mathbb{C}$, Hind H. G. Hashem ${ }^{1,+} \mathbb{C}^{\mathbb{D}}$ and Shorouk M. Al-Issa ${ }^{2,3,+} \mathbb{D}$ \\ 1 Faculty of Science, Alexandria University, Alexandria 21544, Egypt; 3922@qu.edu.sa \\ 2 Department of Mathematics, Faculty of Arts and Sciences, The International University of Beirut, \\ Beirut 1107, Lebanon; shorouk.alissa@liu.edu.lb \\ 3 Department of Mathematics, Faculty of Arts and Sciences, Lebanese International University, \\ Saida 1600, Lebanon \\ * Correspondence: amasayed@alexu.edu.eg \\ + These authors contributed equally to this work.
}

Citation: El-Sayed, A.M.A.; Hashem, H.H.G.; Al-Issa, S.M. Analytical Study of a $\phi$ - Fractional Order Quadratic Functional Integral Equation. Foundations 2022, 2, 167-183. https://doi.org/10.3390/ foundations 2010010

Academic Editor: Sotiris K. Ntouyas

Received: 9 December 2021 Accepted: 12 January 2022

Published: 25 January 2022

Publisher's Note: MDPI stays neutral with regard to jurisdictional claims in published maps and institutional affiliations.

Copyright: (C) 2022 by the authors. Licensee MDPI, Basel, Switzerland. This article is an open access article distributed under the terms and conditions of the Creative Commons Attribution (CC BY) license (https:// creativecommons.org/licenses/by/ $4.0 /)$.

\begin{abstract}
Quadratic integral equations of fractional order have been studied from different views. Here we shall study the existence of continuous solutions of a $\phi$ - fractional-orders quadratic functional integral equation, establish some properties of these solutions and prove the existence of maximal and minimal solutions of that quadratic integral equation. Moreover, we introduce some particular cases to illustrate our results.
\end{abstract}

Keywords: Carathéodory theorem; $\phi$ - fractional integration; quadratic integral equation; continuous solution; maximal and minimal solutions

\section{Introduction}

Quadratic integral equations have gained much attention and many authors studied the existence of solutions for several classes of nonlinear quadratic integral equations (see e.g., [1-11]).

Quadratic integral equations have been appeared in many useful application and problems of the real world. For example, in the theory of radiative transfer, the kinetic theory of gases, the theory of neutron transport, the queuing theory and the traffic theory $[2,5,6,12]$.

In [13], we generalized the Carathéodory theorem for the nonlinear quadratic integral equation

$$
x(t)=a(t)+\int_{0}^{t} f(s, x(s)) d s \int_{0}^{t} g(s, x(s)) d s,
$$

and proved the existence of at least one positive nondecreasing continuous solution to the Equation (1) under the assumption that the functions $f$ and $g$ satisfy the conditions of the Carathèodory Theorem [14]. Furthermore, we proved the existence of the maximal and minimal solutions of the quadratic integral Equation (1).

Let $J=[0, T], \phi_{1}, \phi_{2}: J \rightarrow R$ be increasing and absolutely continuous and $\psi_{i}: J \rightarrow J$, $i=1,2$ be continuous. Let $\alpha, \beta \in(0,1]$ and $t \in J$.

Consider the $\phi-$ fractional-orders quadratic functional integral equation

$$
\begin{aligned}
x(t)= & a(t)+\int_{0}^{t} \frac{\left(\phi_{1}(t)-\phi_{1}(s)\right)^{\alpha-1}}{\Gamma(\alpha)} f_{1}\left(s, x\left(\psi_{1}(s)\right)\right) \phi_{1}^{\prime}(s) d s \\
& \cdot \int_{0}^{t} \frac{\left(\phi_{2}(t)-\phi_{2}(s)\right)^{\beta-1}}{\Gamma(\beta)} f_{2}\left(s, x\left(\psi_{2}(s)\right)\right) \phi_{2}^{\prime}(s) d s, t \in J, \alpha, \beta \in(0,1] .
\end{aligned}
$$

Now, we shall generalize these results and obtain similar ones for the fractional quadratic $\phi$ - integral Equation (2), which in turn gives the existence as well as the existence of many key integral and functional equations that arise in nonlinear analysis and its applications. Finally, we discuss the existence of maximal and minimal solutions of (2). 
Now, we shall denote by $L_{\phi}^{1}=L_{\phi}^{1}[0, T]$ the space of all real functions defined on $J$. such that $\phi^{\prime}(t) f(t) \in L^{1}(J)$ and $\int_{0}^{T}\left|\phi^{\prime}(t) f(t)\right| d t \leq \infty$. Where $\phi$ is an increasing function and absolutely continuous on $J$ and we introduce the norm [9]

$$
\|f(t)\|_{L_{\phi}^{1}}=\int_{0}^{T}\left|\phi^{\prime}(t) f(t)\right| d t \quad t \in J .
$$

Definition 1 ([9]). The $\phi-$ fractional integral of order $\alpha \geq 0$ of the function $f(t) \in L_{\phi}^{1}$ is defined as

$$
I_{\phi}^{\alpha} f(t)=\int_{0}^{t} \frac{(\phi(t)-\phi(s))^{\alpha-1}}{\Gamma(\alpha)} \phi^{\prime}(s) f(s) d s .
$$

$I_{\phi}^{\alpha}$ may be known as the fractional integral of the function $f(t)$ with respect to $\phi(t)$, which is defined for any monotonic increasing function $\phi(t) \geq 0$, with a continuous derivative.

\section{Main Results}

Consider the functional quadratic $\phi-$ integral equation of fractional order (2) under the following assumptions:

(i) $a: J \rightarrow R_{+}$is continuous and $\sup _{t \in J}|a(t)|=k$;

(ii) $f_{1}, f_{2}: J \times R \rightarrow R_{+}$satisfy the Carathéodory condition (i.e., measurable in $t$ for all $x \in R$ and continuous in $x$ for all $t \in J$ ).

(iii) There exist two functions $m_{1}, m_{2} \in L^{1}$ and nonnegative constants $b_{1}, b_{2}$ such that $\left|f_{i}(t, x(t))\right| \leq\left|m_{i}(t)\right|+b_{i}|x|, i=1,2$.

(iv) $\phi_{i}: J \rightarrow R, i=1,2$ are increasing and absolutely continuous.

(v) $\psi_{i}: J \rightarrow J, i=1,2$ are continuous.

(vi) $I_{\phi_{i}}^{\gamma_{i}} m_{i} \leq M_{i}, i=1,2 \forall \gamma_{1} \leq \alpha, \gamma_{2} \leq \beta$.

(vii) $r$ is a positive solution of the inequality:

$$
\begin{aligned}
k & +\frac{M_{1} M_{2} T^{\alpha+\beta-\gamma_{1}-\gamma_{2}}}{\Gamma\left(\alpha-\gamma_{1}+1\right) \Gamma\left(\beta-\gamma_{2}+1\right)}+\frac{b_{1} M_{2} r T^{\alpha+\beta-\gamma_{2}}}{\Gamma(\alpha+1) \Gamma\left(\beta-\gamma_{2}+1\right)} \\
& +\frac{b_{2} M_{1} r T^{\beta+\alpha-\gamma_{1}}}{\Gamma(\beta+1) \Gamma\left(\alpha-\gamma_{1}+1\right)}+\frac{b_{1} b_{2} r^{2} T^{\alpha+\beta}}{\Gamma(\alpha+1) \Gamma(\beta+1)} \leq r .
\end{aligned}
$$

With the aim of proving the existence of at least one solution for the Equation (2), firstly we construct an iterative scheme (as done in the original Carathéodory theorem) and secondly we apply the Schauder fixed point theorem.

\subsection{Existence Results of QFIE (2) via Iterative Scheme}

Theorem 1. Let assumptions (i)-(vii) be satisfied, then the functional quadratic integral equation of fractional order (2) has at least one positive solution $x \in C(J)$.

Proof. Consider the ball $S_{r}$ in the space $C(J)$ defined as

$$
S_{r}=\{x \in C(I):|x(t)| \leq r \text { for } t \in J\} .
$$

Define the sequence $\left\{x_{n}(t)\right\}, t \in\left[0, T-\frac{1}{n}\right]$

$$
\begin{aligned}
x_{n}(t)=a(t) & +\int_{0}^{t+\frac{1}{n}} \frac{\left(\phi_{1}\left(t+\frac{1}{n}\right)-\phi_{1}(s)\right)^{\alpha-1}}{\Gamma(\alpha)} f_{1}\left(s, x_{n}\left(\psi_{1}(s)\right)\right) \phi_{1}^{\prime}(s) d s \\
& \cdot \int_{0}^{t+\frac{1}{n}} \frac{\left(\phi_{2}\left(t+\frac{1}{n}\right)-\phi_{2}(s)\right)^{\beta-1}}{\Gamma(\alpha)} f_{2}\left(s, x_{n}\left(\psi_{2}(s)\right)\right) \phi_{2}^{\prime}(s) d s .
\end{aligned}
$$


The sequence $\left\{x_{n}(t)\right\}, \quad t \in\left[0, T-\frac{1}{n}\right]$ is uniformly bounded

$$
\begin{aligned}
& \left|x_{n}(t)\right| \leq|a(t)|+\int_{0}^{t+\frac{1}{n}} \frac{\left(\phi_{1}\left(t+\frac{1}{n}\right)-\phi_{1}(s)\right)^{\alpha-1}}{\Gamma(\alpha)}\left[\left|m_{1}(s)\right|+b_{1}\left|x_{n}\left(\psi_{1}(s)\right)\right|\right] \phi_{1}^{\prime}(s) d s \\
& \text { - } \int_{0}^{t+\frac{1}{n}} \frac{\left(\phi_{2}\left(t+\frac{1}{n}\right)-\phi_{2}(s)\right)^{\beta-1}}{\Gamma(\alpha)}\left[\left|m_{2}(s)\right|+b_{2}\left|x_{n}\left(\psi_{2}(s)\right)\right|\right] \phi_{2}^{\prime}(s) d s, \\
& \leq|a(t)|+\int_{0}^{t+\frac{1}{n}} \frac{\left(\phi_{1}\left(t+\frac{1}{n}\right)-\phi_{1}(s)\right)^{\alpha-1}}{\Gamma(\alpha)}\left|m_{1}(s)\right| \phi_{1}^{\prime}(s) d s \\
& \text {. } \int_{0}^{t+\frac{1}{n}} \frac{\left(\phi_{2}\left(t+\frac{1}{n}\right)-\phi_{2}(s)\right)^{\beta-1}}{\Gamma(\beta)}\left|m_{2}(s)\right| \phi_{2}^{\prime}(s) d s \\
& +\int_{0}^{t+\frac{1}{n}} \frac{\left(\phi_{1}\left(t+\frac{1}{n}\right)-\phi_{1}(s)\right)^{\alpha-1}}{\Gamma(\alpha)} b_{1}\left|x_{n}\left(\psi_{1}(s)\right)\right| \phi_{1}^{\prime}(s) d s \\
& \text { - } \int_{0}^{t+\frac{1}{n}} \frac{\left(\phi_{2}\left(t+\frac{1}{n}\right)-\phi_{2}(s)\right)^{\beta-1}}{\Gamma(\beta)}\left|m_{2}(s)\right| \phi_{2}^{\prime}(s) d s \\
& +\int_{0}^{t+\frac{1}{n}} \frac{\left(\phi_{2}\left(t+\frac{1}{n}\right)-\phi_{2}(s)\right)^{\beta-1}}{\Gamma(\beta)} b_{2}\left|x_{n}\left(\psi_{2}(s)\right)\right| \phi_{2}^{\prime}(s) d s \\
& \text {. } \int_{0}^{t+\frac{1}{n}} \frac{\left(\phi_{1}\left(t+\frac{1}{n}\right)-\phi_{1}(s)\right)^{\alpha-1}}{\Gamma(\alpha)}\left|m_{1}(s)\right| \phi_{1}^{\prime}(s) d s \\
& +\int_{0}^{t+\frac{1}{n}} \frac{\left(\phi_{1}\left(t+\frac{1}{n}\right)-\phi_{1}(s)\right)^{\alpha-1}}{\Gamma(\alpha)} b_{1}\left|x_{n}\left(\psi_{1}(s)\right)\right| \phi_{1}^{\prime}(s) d s \\
& \text { - } \int_{0}^{t+\frac{1}{n}} \frac{\left(\phi_{2}\left(t+\frac{1}{n}\right)-\phi_{2}(s)\right)^{\beta-1}}{\Gamma(\beta)} b_{2}\left|x_{n}\left(\psi_{2}(s)\right)\right| \phi_{2}^{\prime}(s) d s \\
& \leq k+M_{1} M_{2} \int_{0}^{t+\frac{1}{n}} \frac{\left(\phi_{1}\left(t+\frac{1}{n}\right)-\phi_{1}(s)\right)^{\alpha-\gamma_{1}-1}}{\Gamma\left(\alpha-\gamma_{1}\right)} \phi_{1}^{\prime}(s) d s \\
& \text { - } \int_{0}^{t+\frac{1}{n}} \frac{\left(\phi_{2}\left(t+\frac{1}{n}\right)-\phi_{2}(s)\right)^{\beta-\gamma_{2}-1}}{\Gamma\left(\beta-\gamma_{2}\right)} \phi_{2}^{\prime}(s) d s \\
& +b_{1} M_{2} r \int_{0}^{t+\frac{1}{n}} \frac{\left(\phi_{1}\left(t+\frac{1}{n}\right)-\phi_{1}(s)\right)^{\alpha-1}}{\Gamma(\alpha)} \phi_{1}^{\prime}(s) d s \\
& \cdot \int_{0}^{t+\frac{1}{n}} \frac{\left(\phi_{2}\left(t+\frac{1}{n}\right)-\phi_{2}(s)\right)^{\beta-\gamma_{2}-1}}{\Gamma\left(\beta-\gamma_{2}\right)} \phi_{2}^{\prime}(s) d s \\
& +b_{2} M_{1} r \int_{0}^{t+\frac{1}{n}} \frac{\left(\phi_{1}\left(t+\frac{1}{n}\right)-\phi_{1}(s)\right)^{\alpha-\gamma_{1}-1}}{\Gamma\left(\alpha-\gamma_{1}\right)} \phi_{1}^{\prime}(s) d s \\
& \text { - } \int_{0}^{t+\frac{1}{n}} \frac{\left(\phi_{2}\left(t+\frac{1}{n}\right)-\phi_{2}(s)\right)^{\beta-1}}{\Gamma(\beta)} \mid \phi_{2}^{\prime}(s) d s \\
& +b_{1} b_{2} r^{2} \int_{0}^{t+\frac{1}{n}} \frac{\left(\phi_{1}\left(t+\frac{1}{n}\right)-\phi_{1}(s)\right)^{\alpha-1}}{\Gamma(\alpha)} \phi_{1}^{\prime}(s) d s \\
& \cdot \int_{0}^{t+\frac{1}{n}} \frac{\left(\phi_{2}\left(t+\frac{1}{n}\right)-\phi_{2}(s)\right)^{\beta-1}}{\Gamma(\beta)} \phi_{2}^{\prime}(s) d s \\
& \leq k+M_{1} M_{2} \frac{\left(\phi_{1}\left(t+\frac{1}{n}\right)\right)^{\alpha-\gamma_{1}}}{\Gamma\left(\alpha-\gamma_{1}+1\right)} \frac{\left(\phi_{2}\left(t+\frac{1}{n}\right)\right)^{\beta-\gamma_{2}}}{\Gamma\left(\beta-\gamma_{2}+1\right)} \\
& +\quad b_{1} M_{2} r \frac{\left(\phi_{1}\left(t+\frac{1}{n}\right)\right)^{\alpha}}{\Gamma(\alpha+1)} \cdot \frac{\left(\phi_{2}\left(t+\frac{1}{n}\right)\right)^{\beta-\gamma_{2}}}{\Gamma\left(\beta-\gamma_{2}+1\right)} \\
& +b_{2} M_{1} r \frac{\left(\phi_{2}\left(t+\frac{1}{n}\right)\right)^{\beta}}{\Gamma(\beta+1)} \cdot \frac{\left(\phi_{1}\left(t+\frac{1}{n}\right)\right)^{\alpha-\gamma_{1}}}{\Gamma\left(\alpha-\gamma_{1}+1\right)}+b_{1} b_{2} r^{2} \frac{\left(\phi_{1}\left(t+\frac{1}{n}\right)\right)^{\alpha}}{\Gamma(\alpha+1)} \cdot \frac{\left(\phi_{2}\left(t+\frac{1}{n}\right)\right)^{\beta}}{\Gamma(\beta+1)}
\end{aligned}
$$




$$
\begin{aligned}
& \leq k+\frac{M_{1} M_{2} T^{\alpha-\gamma_{1}}}{\Gamma\left(\alpha-\gamma_{1}+1\right)} \frac{T^{\beta-\gamma_{2}}}{\Gamma\left(\beta-\gamma_{2}+1\right)}+\frac{b_{1} M_{2} r T^{\alpha}}{\Gamma(\alpha+1)} \cdot \frac{T^{\beta-\gamma_{2}}}{\Gamma\left(\beta-\gamma_{2}+1\right)} \\
& +\frac{b_{2} M_{1} r T^{\beta}}{\Gamma(\beta+1)} \cdot \frac{T^{\alpha-\gamma_{1}}}{\Gamma\left(\alpha-\gamma_{1}+1\right)}+\frac{b_{1} b_{2} r^{2} T^{\alpha}}{\Gamma(\alpha+1)} \cdot \frac{T^{\beta}}{\Gamma(\beta+1)} \\
& \leq k+\frac{M_{1} M_{2} T^{\alpha+\beta-\gamma_{1}-\gamma_{2}}}{\Gamma\left(\alpha-\gamma_{1}+1\right) \Gamma\left(\beta-\gamma_{2}+1\right)}+\frac{b_{1} M_{2} r T^{\alpha+\beta-\gamma_{2}}}{\Gamma(\alpha+1) \Gamma\left(\beta-\gamma_{2}+1\right)} \\
& +\frac{b_{2} M_{1} r T^{\beta+\alpha-\gamma_{1}}}{\Gamma(\beta+1) \Gamma\left(\alpha-\gamma_{1}+1\right)}+\frac{b_{1} b_{2} r^{2} T^{\alpha+\beta}}{\Gamma(\alpha+1) \Gamma(\beta+1)} \leq r .
\end{aligned}
$$

Also, the sequence is equi-continuous.

For $t_{1}, t_{2} \in\left[0, T-\frac{1}{n}\right]$ such that $t_{1}<t_{2}$, we have

$$
\begin{aligned}
&\left|x_{n}\left(t_{2}\right)-x_{n}\left(t_{1}\right)\right|=\mid a\left(t_{2}\right)-a\left(t_{1}\right) \\
&+\int_{0}^{t_{2}+\frac{1}{n}} \frac{\left(\phi_{1}\left(t_{2}+\frac{1}{n}\right)-\phi_{1}(s)\right)^{\alpha-1}}{\Gamma(\alpha)} f_{1}\left(s, x_{n}\left(\psi_{1}(s)\right)\right) \phi_{1}^{\prime}(s) d s \\
& \cdot \int_{0}^{t_{2}+\frac{1}{n}} \frac{\left(\phi_{2}\left(t_{2}+\frac{1}{n}\right)-\phi_{2}(s)\right)^{\beta-1}}{\Gamma(\beta)} f_{2}\left(s, x_{n}\left(\psi_{2}(s)\right)\right) \phi_{2}^{\prime}(s) d s \\
&-\int_{0}^{t_{1}+\frac{1}{n}} \frac{\left(\phi_{1}\left(t_{1}+\frac{1}{n}\right)-\phi_{1}(s)\right)^{\alpha-1}}{\Gamma(\alpha)} f_{1}\left(s, x_{n}\left(\psi_{1}(s)\right)\right) \phi_{1}^{\prime}(s) d s \\
& \cdot \int_{0}^{t_{1}+\frac{1}{n}} \frac{\left(\phi_{2}\left(t_{1}+\frac{1}{n}\right)-\phi_{2}(s)\right)^{\beta-1}}{\Gamma(\beta)} f_{2}\left(s, x_{n}\left(\psi_{2}(s)\right)\right) \phi_{2}^{\prime}(s) d s \mid \\
&=\mid a\left(t_{2}\right)-a\left(t_{1}\right) \\
&+\int_{0}^{t_{1}+\frac{1}{n}} \frac{\left(\phi_{1}\left(t_{2}+\frac{1}{n}\right)-\phi_{1}(s)\right)^{\alpha-1}}{\Gamma(\alpha)} f_{1}\left(s, x_{n}\left(\psi_{1}(s)\right)\right) \phi_{1}^{\prime}(s) d s \\
& \cdot \int_{0}^{t_{2}+\frac{1}{n}} \frac{\left(\phi_{2}\left(t_{2}+\frac{1}{n}\right)-\phi_{2}(s)\right)^{\beta-1}}{\Gamma(\beta)} f_{2}\left(s, x_{n}\left(\psi_{2}(s)\right)\right) \phi_{2}^{\prime}(s) d s \\
&+\int_{t_{1}+\frac{1}{n}}^{t_{2}+\frac{1}{n}} \frac{\left(\phi_{1}\left(t_{2}+\frac{1}{n}\right)-\phi_{1}(s)\right)^{\alpha-1}}{\Gamma(\alpha)} f_{1}\left(s, x_{n}\left(\psi_{1}(s)\right)\right) \phi_{1}^{\prime}(s) d s \\
& \cdot \int_{0}^{t_{2}+\frac{1}{n}} \frac{\left(\phi_{2}\left(t_{2}+\frac{1}{n}\right)-\phi_{2}(s)\right)^{\beta-1}}{\Gamma(\beta)} f_{2}\left(s, x_{n}\left(\psi_{2}(s)\right)\right) \phi_{2}^{\prime}(s) d s \\
&-\int_{0}^{t_{1}+\frac{1}{n}} \frac{\left(\phi_{1}\left(t_{1}+\frac{1}{n}\right)-\phi_{1}(s)\right)^{\alpha-1}}{\Gamma(\alpha)} f_{1}\left(s, x_{n}\left(\psi_{1}(s)\right)\right) \phi_{1}^{\prime}(s) d s \\
& \cdot \int_{0}^{t_{1}+\frac{1}{n}} \frac{\left(\phi_{2}\left(t_{1}+\frac{1}{n}\right)-\phi_{2}(s)\right)^{\beta-1}}{\Gamma(\beta)} f_{2}\left(s, x_{n}\left(\psi_{2}(s)\right)\right) \phi_{2}^{\prime}(s) d s \mid
\end{aligned}
$$




$$
\begin{aligned}
& \leq \mid a\left(t_{2}\right)-a\left(t_{1}\right) \\
& +\int_{0}^{t_{1}+\frac{1}{n}} \frac{\left(\phi_{1}\left(t_{2}+\frac{1}{n}\right)-\phi_{1}(s)\right)^{\alpha-1}}{\Gamma(\alpha)} f_{1}\left(s, x_{n}\left(\psi_{1}(s)\right)\right) \phi_{1}^{\prime}(s) d s \\
& \text { - } \int_{0}^{t_{1}+\frac{1}{n}} \frac{\left(\phi_{2}\left(t_{2}+\frac{1}{n}\right)-\phi_{2}(s)\right)^{\beta-1}}{\Gamma(\beta)} f_{2}\left(s, x_{n}\left(\psi_{2}(s)\right)\right) \phi_{2}^{\prime}(s) d s \\
& +\int_{0}^{t_{1}+\frac{1}{n}} \frac{\left(\phi_{1}\left(t_{2}+\frac{1}{n}\right)-\phi_{1}(s)\right)^{\alpha-1}}{\Gamma(\alpha)} f_{1}\left(s, x_{n}\left(\psi_{1}(s)\right)\right) \phi_{1}^{\prime}(s) d s \\
& \text { - } \quad \int_{t_{1}+\frac{1}{n}}^{t_{2}+\frac{1}{n}} \frac{\left(\phi_{2}\left(t_{2}+\frac{1}{n}\right)-\phi_{2}(s)\right)^{\beta-1}}{\Gamma(\beta)} f_{2}\left(s, x_{n}\left(\psi_{2}(s)\right)\right) \phi_{2}^{\prime}(s) d s \\
& +\int_{t_{1}+\frac{1}{n}}^{t_{2}+\frac{1}{n}} \frac{\left(\phi_{1}\left(t_{2}+\frac{1}{n}\right)-\phi_{1}(s)\right)^{\alpha-1}}{\Gamma(\alpha)} f_{1}\left(s, x_{n}\left(\psi_{1}(s)\right)\right) \phi_{1}^{\prime}(s) d s \\
& \text { - } \int_{0}^{t_{2}+\frac{1}{n}} \frac{\left(\phi_{2}\left(t_{1}+\frac{1}{n}\right)-\phi_{2}(s)\right)^{\beta-1}}{\Gamma(\beta)} f_{2}\left(s, x_{n}\left(\psi_{2}(s)\right)\right) \phi_{2}^{\prime}(s) d s \\
& -\int_{0}^{t_{1}+\frac{1}{n}} \frac{\left(\phi_{1}\left(t_{1}+\frac{1}{n}\right)-\phi_{1}(s)\right)^{\alpha-1}}{\Gamma(\alpha)} f_{1}\left(s, x_{n}\left(\psi_{1}(s)\right)\right) \phi_{1}^{\prime}(s) d s \\
& \text { - } \int_{0}^{t_{1}+\frac{1}{n}} \frac{\left(\phi_{2}\left(t_{1}+\frac{1}{n}\right)-\phi_{2}(s)\right)^{\beta-1}}{\Gamma(\beta)} f_{2}\left(s, x_{n}\left(\psi_{2}(s)\right)\right) \phi_{2}^{\prime}(s) d s \mid \\
& \leq \mid a\left(t_{2}\right)-a\left(t_{1}\right) \\
& +\int_{0}^{t_{1}+\frac{1}{n}} \frac{\left[\left(\phi_{1}\left(t_{2}+\frac{1}{n}\right)-\phi_{1}(s)\right)^{\alpha-1}-\left(\phi_{1}\left(t_{1}+\frac{1}{n}\right)-\phi_{1}(s)\right)^{\alpha-1}\right]}{\Gamma(\alpha)} f_{1}\left(s, x_{n}\left(\psi_{1}(s)\right)\right) \phi_{1}^{\prime}(s) d s \\
& \text { - } \int_{0}^{t_{1}+\frac{1}{n}} \frac{\left(\phi_{2}\left(t_{2}+\frac{1}{n}\right)-\phi_{2}(s)\right)^{\beta-1}}{\Gamma(\beta)} f_{2}\left(s, x_{n}\left(\psi_{2}(s)\right)\right) \phi_{2}^{\prime}(s) d s \\
& +\int_{0}^{t_{1}+\frac{1}{n}} \frac{\left[\left(\phi_{1}\left(t_{2}+\frac{1}{n}\right)-\phi_{1}(s)\right)^{\alpha-1}-\left(\phi_{1}\left(t_{1}+\frac{1}{n}\right)-\phi_{1}(s)\right)^{\alpha-1}\right]}{\Gamma(\alpha)} f_{1}\left(s, x_{n}\left(\psi_{1}(s)\right)\right) \phi_{1}^{\prime}(s) d s \\
& \cdot \int_{0}^{t_{1}+\frac{1}{n}} \frac{\left(\phi_{2}\left(t_{1}+\frac{1}{n}\right)-\phi_{2}(s)\right)^{\beta-1}}{\Gamma(\beta)} f_{2}\left(s, x_{n}\left(\psi_{2}(s)\right)\right) \phi_{2}^{\prime}(s) d s \\
& +\int_{0}^{t_{1}+\frac{1}{n}} \frac{\left(\phi_{1}\left(t_{2}+\frac{1}{n}\right)-\phi_{1}(s)\right)^{\alpha-1}}{\Gamma(\alpha)} f_{1}\left(s, x_{n}\left(\psi_{1}(s)\right)\right) \phi_{1}^{\prime}(s) d s \\
& \text { - } \quad \int_{t_{1}+\frac{1}{n}}^{t_{2}+\frac{1}{n}} \frac{\left(\phi_{2}\left(t_{2}+\frac{1}{n}\right)-\phi_{2}(s)\right)^{\beta-1}}{\Gamma(\beta)} f_{2}\left(s, x_{n}\left(\psi_{2}(s)\right)\right) \phi_{2}^{\prime}(s) d s \\
& +\int_{t_{1}+\frac{1}{n}}^{t_{2}+\frac{1}{n}} \frac{\left(\phi_{1}\left(t_{2}+\frac{1}{n}\right)-\phi_{1}(s)\right)^{\alpha-1}}{\Gamma(\alpha)} f_{1}\left(s, x_{n}\left(\psi_{1}(s)\right)\right) \phi_{1}^{\prime}(s) d s \\
& \text { - } \int_{0}^{t_{2}+\frac{1}{n}} \frac{\left(\phi_{2}\left(t_{1}+\frac{1}{n}\right)-\phi_{2}(s)\right)^{\beta-1}}{\Gamma(\beta)} f_{2}\left(s, x_{n}\left(\psi_{2}(s)\right)\right) \phi_{2}^{\prime}(s) d s \mid \\
& \leq\left|a\left(t_{2}\right)-a\left(t_{1}\right)\right| \\
& +\int_{0}^{t_{1}+\frac{1}{n}} \frac{\left[\left(\phi_{1}\left(t_{2}+\frac{1}{n}\right)-\phi_{1}(s)\right)^{\alpha-1}-\left(\phi_{1}\left(t_{1}+\frac{1}{n}\right)-\phi_{1}(s)\right)^{\alpha-1}\right]}{\Gamma(\alpha)}\left|f_{1}\left(s, x_{n}\left(\psi_{1}(s)\right)\right)\right| \phi_{1}^{\prime}(s) d s \\
& \cdot \int_{0}^{t_{1}+\frac{1}{n}} \frac{\left(\phi_{2}\left(t_{2}+\frac{1}{n}\right)-\phi_{2}(s)\right)^{\beta-1}}{\Gamma(\beta)}\left|f_{2}\left(s, x_{n}\left(\psi_{2}(s)\right)\right)\right| \phi_{2}^{\prime}(s) d s \\
& +\int_{0}^{t_{1}+\frac{1}{n}} \frac{\left[\left(\phi_{1}\left(t_{2}+\frac{1}{n}\right)-\phi_{1}(s)\right)^{\alpha-1}-\left(\phi_{1}\left(t_{1}+\frac{1}{n}\right)-\phi_{1}(s)\right)^{\alpha-1}\right]}{\Gamma(\alpha)}\left|f_{1}\left(s, x_{n}\left(\psi_{1}(s)\right)\right)\right| \phi_{1}^{\prime}(s) d s \\
& \cdot \int_{0}^{t_{1}+\frac{1}{n}} \frac{\left(\phi_{2}\left(t_{1}+\frac{1}{n}\right)-\phi_{2}(s)\right)^{\beta-1}}{\Gamma(\beta)}\left|f_{2}\left(s, x_{n}\left(\psi_{2}(s)\right)\right)\right| \phi_{2}^{\prime}(s) d s
\end{aligned}
$$




$$
\begin{array}{ll}
+ & \int_{0}^{t_{1}+\frac{1}{n}} \frac{\left(\phi_{1}\left(t_{2}+\frac{1}{n}\right)-\phi_{1}(s)\right)^{\alpha-1}}{\Gamma(\alpha)}\left|f_{1}\left(s, x_{n}\left(\psi_{1}(s)\right)\right) \phi_{1}^{\prime}(s)\right| d s \\
& \cdot \quad \int_{t_{1}+\frac{1}{n}}^{t_{2}+\frac{1}{n}} \frac{\left(\phi_{2}\left(t_{2}+\frac{1}{n}\right)-\phi_{2}(s)\right)^{\beta-1}}{\Gamma(\beta)}\left|f_{2}\left(s, x_{n}\left(\psi_{2}(s)\right)\right)\right| \phi_{2}^{\prime}(s) d s \\
+ & \int_{t_{1}+\frac{1}{n}}^{t_{2}+\frac{1}{n}} \frac{\left(\phi_{1}\left(t_{2}+\frac{1}{n}\right)-\phi_{1}(s)\right)^{\alpha-1}}{\Gamma(\alpha)}\left|f_{1}\left(s, x_{n}\left(\psi_{1}(s)\right)\right)\right| \phi_{1}^{\prime}(s) d s \\
& \int_{0}^{t_{2}+\frac{1}{n}} \frac{\left(\phi_{2}\left(t_{1}+\frac{1}{n}\right)-\phi_{2}(s)\right)^{\beta-1}}{\Gamma(\beta)}\left|f_{2}\left(s, x_{n}\left(\psi_{2}(s)\right)\right)\right| \phi_{2}^{\prime}(s) d s \\
+ & \int_{t_{1}+\frac{1}{n}}^{t_{2}+\frac{1}{n}} \frac{\left(\phi_{1}\left(t_{1}+\frac{1}{n}\right)-\phi_{1}(s)\right)^{\alpha-1}}{\Gamma(\alpha)}\left|f_{1}\left(s, x_{n}\left(\psi_{1}(s)\right)\right)\right| \phi_{1}^{\prime}(s) d s \\
& \int_{t_{1}+\frac{1}{n}}^{t_{2}+\frac{1}{n}} \frac{\left(\phi_{2}\left(t_{1}+\frac{1}{n}\right)-\phi_{2}(s)\right)^{\beta-1}}{\Gamma(\beta)}\left|f_{2}\left(s, x_{n}\left(\psi_{2}(s)\right)\right)\right| \phi_{2}^{\prime}(s) d s .
\end{array}
$$

Then we obtain

$$
\begin{aligned}
& \left|x_{n}\left(t_{2}\right)-x_{n}\left(t_{1}\right)\right| \leq\left|a\left(t_{2}\right)-a\left(t_{1}\right)\right| \\
+ & \int_{0}^{t_{1}+\frac{1}{n}} \frac{\left[\left(\phi_{1}\left(t_{2}+\frac{1}{n}\right)-\phi_{1}(s)\right)^{\alpha-1}-\left(\phi_{1}\left(t_{1}+\frac{1}{n}\right)-\phi_{1}(s)\right)^{\alpha-1}\right]}{\Gamma(\alpha)}\left[m_{1}(s)+b_{1} r\right] \phi_{1}^{\prime}(s) d s \\
\cdot & \int_{0}^{t_{1}+\frac{1}{n}} \frac{\left[\left(\phi_{2}\left(t_{2}+\frac{1}{n}\right)-\phi_{2}(s)\right)^{\beta-1}-\left(\phi_{2}\left(t_{1}+\frac{1}{n}\right)-\phi_{2}(s)\right)^{\beta-1}\right]}{\Gamma(\beta)}\left[m_{2}(s)+b_{2} r\right] \phi_{2}^{\prime}(s) d s \\
+ & {\left[\frac{M_{1} T^{\alpha-\gamma_{1}}}{\Gamma\left(\alpha-\gamma_{1}+1\right)}+\frac{b_{1} r T^{\alpha}}{\Gamma(\alpha+1)}\right] } \\
\cdot & {\left[\frac{M_{2}\left(\phi_{2}\left(t_{1}+\frac{1}{n}\right)-\phi_{2}\left(t+\frac{1}{n}\right)^{\beta-\gamma_{2}}\right.}{\Gamma\left(\beta-\gamma_{2}+1\right)}+\frac{b_{2} r\left(\phi_{2}\left(t_{1}+\frac{1}{n}\right)-\phi_{2}\left(t_{2}+\frac{1}{n}\right)\right)^{\beta}}{\Gamma(\beta+1)}\right] } \\
+ & {\left[\frac{M_{1}\left(\phi_{1}\left(t_{1}+\frac{1}{n}\right)-\phi_{1}\left(t_{2}+\frac{1}{n}\right)\right)^{\alpha-\gamma_{1}}}{\Gamma\left(\alpha-\gamma_{1}+1\right)}+\frac{b_{1} r\left(\phi_{1}\left(t_{1}+\frac{1}{n}\right)-\phi_{1}\left(t_{2}+\frac{1}{n}\right)\right)^{\alpha}}{\Gamma(\alpha+1)}\right] } \\
\cdot & {\left[\frac{M_{2}\left(\phi_{2}\left(t_{1}+\frac{1}{n}\right)-\phi_{2}\left(t_{2}+\frac{1}{n}\right)\right)^{\beta-\gamma_{2}}}{\Gamma\left(\beta-\gamma_{2}+1\right)}+\frac{b_{2} r\left(\phi_{2}\left(t_{1}+\frac{1}{n}\right)-\phi_{2}\left(t_{2}+\frac{1}{n}\right)\right)^{\beta}}{\Gamma(\beta+1)}\right] } \\
+ & {\left[\frac{M_{1}\left(\phi_{1}\left(t_{1}+\frac{1}{n}\right)-\phi_{1}\left(t_{2}+\frac{1}{n}\right)\right)^{\alpha-\gamma_{1}}}{\Gamma\left(\alpha-\gamma_{1}+1\right)}+\frac{b_{1} r\left(\phi_{1}\left(t_{1}+\frac{1}{n}\right)-\phi_{1}\left(t_{2}+\frac{1}{n}\right)\right)^{\alpha}}{\Gamma(\alpha+1)}\right] . } \\
\cdot & {\left[\frac{M_{2}\left(\phi_{2}\left(t_{1}+\frac{1}{n}\right)-\phi_{2}\left(t_{2}\right)+\frac{1}{n}\right)^{\beta-\gamma_{2}}}{\Gamma\left(\beta-\gamma_{2}+1\right)}+\frac{b_{2} r\left(\phi_{2}\left(t_{1}+\frac{1}{n}\right)-\phi_{2}\left(t_{2}+\frac{1}{n}\right)\right)^{\beta}}{\Gamma(\beta+1)}\right] . }
\end{aligned}
$$

This implies

$$
\left|t_{2}-t_{1}\right| \rightarrow 0 \Rightarrow\left|x_{n}\left(t_{2}\right)-x_{n}\left(t_{1}\right)\right| \rightarrow 0
$$

and this proves the equi-continuity of the sequence $\left\{x_{n}(t)\right\}$. Hence, $\left\{x_{n}(t)\right\}$ is a sequence of equi-continuous and uniformly bounded functions.

By Arzela-Ascoli Theorem [14], then there exists a subsequence $\left\{x_{n_{k}}(t)\right\}$ of continuous functions which converges uniformly to a continuous function $x$ as $k \rightarrow \infty$.

Now we show that this limit function is the required solution.

From assumptions (ii) and (iii) we have

$$
\left|f_{i}\left(s, x_{n_{k}}\left(\psi_{1}(s)\right)\right)\right| \leq\left|m_{i}(s)\right|+b_{i} r \in L^{1},
$$

and the functions $f_{i}\left(s, x_{n_{k}}\left(\psi_{i}(s)\right)\right), i=1,2$ are continuous in the second argument,

$$
\text { i.e., } f_{i}\left(s, x_{n_{k}}\left(\psi_{i}(s)\right)\right) \rightarrow f_{i}\left(s, x\left(\psi_{i}(s)\right)\right) \text { as } k \rightarrow \infty \text {. }
$$


For $s \in(0, t)$ and $t \in J$

$\left(\phi_{1}\left(t+\frac{1}{n_{k}}\right)-\phi_{1}(s)\right)>\left(\phi_{1}(t)-\phi_{1}(s)\right) \Rightarrow\left(\phi_{1}\left(t+\frac{1}{n_{k}}\right)-\phi_{1}(s)\right)^{\alpha-1}<\left(\phi_{1}(t)-\phi_{1}(s)\right)^{\alpha-1}$,

and

$\left(\phi_{2}\left(t+\frac{1}{n_{k}}\right)-\phi_{2}(s)\right)>\left(\phi_{2}(t)-\phi_{2}(s)\right) \Rightarrow\left(\phi_{2}\left(t+\frac{1}{n_{k}}\right)-\phi_{2}(s)\right)^{\beta-1}<\left(\phi_{2}(t)-\phi_{2}(s)\right)^{\beta-1}$,

therefore the sequences $\left\{\left(\phi_{1}\left(t+\frac{1}{n_{k}}\right)-\phi_{1}(s)\right)^{\alpha-1} f_{1}\left(s, x_{n_{k}}\left(\psi_{1}(s)\right)\right)\right\}$,

$\left(\phi_{2}\left(t+\frac{1}{n_{k}}\right)-\phi_{2}(s)\right)^{\beta-1} f_{2}\left(s, x_{n_{k}}\left(\psi_{2}(s)\right)\right), \alpha, \beta \in(0,1]$ satisfy the Lebesgue dominated convergence theorem [14].

$$
\begin{aligned}
& \int_{0}^{t+\frac{1}{n}} \frac{\left(\phi_{1}\left(t+\frac{1}{n}\right)-\phi_{1}(s)\right)^{\alpha-1}}{\Gamma(\alpha)} f_{1}\left(s, x_{n}\left(\psi_{1}(s)\right)\right) \phi_{1}^{\prime}(s) d s \\
\cdot & \int_{0}^{t+\frac{1}{n}} \frac{\left(\phi_{2}\left(t+\frac{1}{n}\right)-\phi_{2}(s)\right)^{\beta-1}}{\Gamma(\alpha)} f_{2}\left(s, x_{n}\left(\psi_{2}(s)\right)\right) \phi_{2}^{\prime}(s) d s \\
\Rightarrow \quad & \int_{0}^{t} \frac{\left(\phi_{1}(t)-\phi_{1}(s)\right)^{\alpha-1}}{\Gamma(\alpha)} f_{1}\left(s, x\left(\psi_{1}(s)\right)\right) \phi_{1}^{\prime}(s) d s \\
& \int_{0}^{t} \frac{\left(\phi_{2}(t)-\phi_{2}(s)\right)^{\beta-1}}{\Gamma(\alpha)} f_{2}\left(s, x\left(\psi_{2}(s)\right)\right) \phi_{2}^{\prime}(s) d s .
\end{aligned}
$$

Similarly we have

$$
\begin{aligned}
x(t) & =\lim _{k \rightarrow \infty} x_{n_{k}}(t)=a(t) \\
& +\int_{0}^{t} \frac{\left(\phi_{1}(t)-\phi_{1}(s)\right)^{\alpha-1}}{\Gamma(\alpha)} f_{1}\left(s, x\left(\psi_{1}(s)\right)\right) \phi_{1}^{\prime}(s) d s \\
& \cdot \int_{0}^{t} \frac{\left(\phi_{2}(t)-\phi_{2}(s)\right)^{\beta-1}}{\Gamma(\alpha)} f_{2}\left(s, x\left(\psi_{2}(s)\right)\right) \phi_{2}^{\prime}(s) d s .
\end{aligned}
$$

which proves the existence of a positive solution $x \in C(J)$ of the quadratic integral Equation (2).

\subsection{Existence Results of QFIE (2) via the Fixed Point Theorem}

In this subsection, we shall prove another existence result for the functional quadratic $\phi-$ integral of fractional order (2) by applying the Schauder fixed point.

Theorem 2. Let assumptions (i)-(vii) hold. Then the $\phi$-fractional-orders quadratic functional integral Equation (2) has at least one solution $x \in C(J)$.

Proof. Fix a number $r>0$ and the ball $S_{r}$ in the space $C(J)$ as defined above.

Let $\mathbb{T}$ be the operator defined on $S_{r}$ by the formula

$$
\begin{aligned}
(\mathbb{T} x)(t)= & a(t)+\int_{0}^{t} \frac{\left(\phi_{1}(t)-\phi_{1}(s)\right)^{\alpha-1}}{\Gamma(\alpha)} f_{1}\left(s, x\left(\psi_{1}(s)\right)\right) \phi_{1}^{\prime}(s) d s \\
& \cdot \int_{0}^{t} \frac{\left(\phi_{2}(t)-\phi_{2}(s)\right)^{\beta-1}}{\Gamma(\beta)} f_{2}\left(s, x\left(\psi_{2}(s)\right)\right) \phi_{2}^{\prime}(s) d s, x \in S_{r}, t \in J .
\end{aligned}
$$

Then, in view of our assumptions, for $x \in S_{r}$ and $t \in J$ we obtain 


$$
\begin{aligned}
& |\mathbb{T} x(t)| \leq|a(t)|+\int_{0}^{t} \frac{\left(\phi_{1}(t)-\phi_{1}(s)\right)^{\alpha-1}}{\Gamma(\alpha)}\left[m_{1}(s)+b_{1}\left|x\left(\psi_{1}(s)\right)\right|\right] \phi_{1}^{\prime}(s) d s \\
& \text { - } \int_{0}^{t} \frac{\left(\phi_{2}(t)-\phi_{2}(s)\right)^{\beta-1}}{\Gamma(\alpha)}\left[m_{2}(s)+b_{2}\left|x\left(\psi_{2}(s)\right)\right|\right] \phi_{2}^{\prime}(s) d s, \\
& \leq|a(t)|+\int_{0}^{t} \frac{\left(\phi_{1}(t)-\phi_{1}(s)\right)^{\alpha-1}}{\Gamma(\alpha)}\left|m_{1}(s)\right| \phi_{1}^{\prime}(s) d s \int_{0}^{t} \frac{\left(\phi_{2}(t)-\phi_{2}(s)\right)^{\beta-1}}{\Gamma(\beta)}\left|m_{2}(s)\right| \phi_{2}^{\prime}(s) d s \\
& +\int_{0}^{t} \frac{\left(\phi_{1}(t)-\phi_{1}(s)\right)^{\alpha-1}}{\Gamma(\alpha)}\left|b_{1}\right| x\left(\psi_{1}(s)\right)\left|\phi_{1}^{\prime}(s) d s . \int_{0}^{t} \frac{\left(\phi_{2}(t)-\phi_{2}(s)\right)^{\beta-1}}{\Gamma(\beta)}\right| m_{2}(s) \mid \phi_{2}^{\prime}(s) d s \\
& +\int_{0}^{t} \frac{\left(\phi_{2}(t)-\phi_{2}(s)\right)^{\beta-1}}{\Gamma(\beta)}\left|b_{2}\right| x\left(\psi_{2}(s)\right)\left|\phi_{2}^{\prime}(s) d s . \int_{0}^{t} \frac{\left(\phi_{1}(t)-\phi_{1}(s)\right)^{\alpha-1}}{\Gamma(\alpha)}\right| m_{1}(s) \mid \phi_{1}^{\prime}(s) d s \\
& +\int_{0}^{t} \frac{\left(\phi_{1}(t)-\phi_{1}(s)\right)^{\alpha-1}}{\Gamma(\alpha)}\left|b_{1}\right| x\left(\psi_{1}(s)\right)\left|\phi_{1}^{\prime}(s) d s . \int_{0}^{t} \frac{\left(\phi_{2}(t)-\phi_{2}(s)\right)^{\beta-1}}{\Gamma(\beta)}\right| b_{2}\left|x\left(\psi_{2}(s)\right)\right| \phi_{2}^{\prime}(s) d s \\
& \leq k+M_{1} M_{2} \int_{0}^{t} \frac{\left(\phi_{1}(t)-\phi_{1}(s)\right)^{\alpha-\gamma_{1}-1}}{\Gamma\left(\alpha-\gamma_{1}\right)} \phi_{1}^{\prime}(s) d s \int_{0}^{t} \frac{\left(\phi_{2}(t)-\phi_{2}(s)\right)^{\beta-\gamma_{2}-1}}{\Gamma\left(\beta-\gamma_{2}\right)} \phi_{2}^{\prime}(s) d s \\
& +b_{1} M_{2} r \int_{0}^{t} \frac{\left(\phi_{1}(t)-\phi_{1}(s)\right)^{\alpha-1}}{\Gamma(\alpha)} \phi_{1}^{\prime}(s) d s . \int_{0}^{t} \frac{\left(\phi_{2}(t)-\phi_{2}(s)\right)^{\beta-\gamma_{2}-1}}{\Gamma\left(\beta-\gamma_{2}\right)} \phi_{2}^{\prime}(s) d s \\
& +\quad b_{2} M_{1} r \int_{0}^{t} \frac{\left(\phi_{2}(t)-\phi_{2}(s)\right)^{\beta-1}}{\Gamma(\beta)} \mid \phi_{2}^{\prime}(s) d s . \int_{0}^{t} \frac{\left(\phi_{1}(t)-\phi_{1}(s)\right)^{\alpha-\gamma_{1}-1}}{\Gamma\left(\alpha-\gamma_{1}\right)} \phi_{1}^{\prime}(s) d s \\
& +b_{1} b_{2} r^{2} \int_{0}^{t} \frac{\left(\phi_{1}(t)-\phi_{1}(s)\right)^{\alpha-1}}{\Gamma(\alpha)} \phi_{1}^{\prime}(s) d s . \int_{0}^{t} \frac{\left(\phi_{2}(t)-\phi_{2}(s)\right)^{\beta-1}}{\Gamma(\beta)} \phi_{2}^{\prime}(s) d s \\
& \leq \quad k+M_{1} M_{2} \frac{\left(\phi_{1}(t)\right)^{\alpha-\gamma_{1}}}{\Gamma\left(\alpha-\gamma_{1}+1\right)} \frac{\left(\phi_{2}(t)\right)^{\beta-\gamma_{2}}}{\Gamma\left(\beta-\gamma_{2}+1\right)}+b_{1} M_{2} r \frac{\left(\phi_{1}(t)\right)^{\alpha}}{\Gamma(\alpha+1)} \cdot \frac{\left(\phi_{2}(t)\right)^{\beta-\gamma_{2}}}{\Gamma\left(\beta-\gamma_{2}+1\right)} \\
& +b_{2} M_{1} r \frac{\left(\phi_{2}(t)\right)^{\beta}}{\Gamma(\beta+1)} \cdot \frac{\left(\phi_{1}(t)\right)^{\alpha-\gamma_{1}}}{\Gamma\left(\alpha-\gamma_{1}+1\right)}+b_{1} b_{2} r^{2} \frac{\left(\phi_{1}(t)\right)^{\alpha}}{\Gamma(\alpha+1)} \cdot \frac{\left(\phi_{2}(t)\right)^{\beta}}{\Gamma(\beta+1)} \\
& \leq \frac{k+M_{1} M_{2} T^{\alpha-\gamma_{1}}}{\Gamma\left(\alpha-\gamma_{1}+1\right)} \frac{T^{\beta-\gamma_{2}}}{\Gamma\left(\beta-\gamma_{2}+1\right)}+\frac{b_{1} M_{2} r T^{\alpha}}{\Gamma(\alpha+1)} \cdot \frac{T^{\beta-\gamma_{2}}}{\Gamma\left(\beta-\gamma_{2}+1\right)} \\
& +\frac{b_{2} M_{1} r T^{\beta}}{\Gamma(\beta+1)} \cdot \frac{T^{\alpha-\gamma_{1}}}{\Gamma\left(\alpha-\gamma_{1}+1\right)}+\frac{b_{1} b_{2} r^{2} T^{\alpha}}{\Gamma(\alpha+1)} \cdot \frac{T^{\beta}}{\Gamma(\beta+1)} \\
& \leq k+\frac{M_{1} M_{2} T^{\alpha+\beta-\gamma_{1}-\gamma_{2}}}{\Gamma\left(\alpha-\gamma_{1}+1\right) \Gamma\left(\beta-\gamma_{2}+1\right)}+\frac{b_{1} M_{2} r T^{\alpha+\beta-\gamma_{2}}}{\Gamma(\alpha+1) \Gamma\left(\beta-\gamma_{2}+1\right)} \\
& +\frac{b_{2} M_{1} r T^{\beta+\alpha-\gamma_{1}}}{\Gamma(\beta+1) \Gamma\left(\alpha-\gamma_{1}+1\right)}+\frac{b_{1} b_{2} r^{2} T^{\alpha+\beta}}{\Gamma(\alpha+1) \Gamma(\beta+1)} \leq r .
\end{aligned}
$$

Hence, in view of the assumption (vii), we have that $\mathbb{T}$ transforms the ball $S_{r}$ into itself. 
Now, for $t_{1}$ and $t_{2} \in I$ (without loss of generality assume that $t_{1}<t_{2}$ ), we have

$$
\begin{aligned}
& \left|(\mathbb{T} x)\left(t_{2}\right)-(\mathbb{T} x)\left(t_{1}\right)\right|=\mid a\left(t_{2}\right)-a\left(t_{1}\right) \\
& +\int_{0}^{t_{2}} \frac{\left(\phi_{1}\left(t_{2}\right)-\phi_{1}(s)\right)^{\alpha-1}}{\Gamma(\alpha)} f_{1}\left(s, x\left(\psi_{1}(s)\right)\right) \phi_{1}^{\prime}(s) d s \\
& \text { - } \quad \int_{0}^{t_{2}} \frac{\left(\phi_{2}\left(t_{2}\right)-\phi_{2}(s)\right)^{\beta-1}}{\Gamma(\beta)} f_{2}\left(s, x\left(\psi_{2}(s)\right)\right) \phi_{2}^{\prime}(s) d s \\
& -\int_{0}^{t_{1}} \frac{\left(\phi_{1}\left(t_{1}\right)-\phi_{1}(s)\right)^{\alpha-1}}{\Gamma(\alpha)} f_{1}\left(s, x\left(\psi_{1}(s)\right)\right) \phi_{1}^{\prime}(s) d s \\
& \text { - } \int_{0}^{t_{1}} \frac{\left(\phi_{2}\left(t_{1}\right)-\phi_{2}(s)\right)^{\beta-1}}{\Gamma(\beta)} f_{2}\left(s, x\left(\psi_{2}(s)\right)\right) \phi_{2}^{\prime}(s) d s \mid \\
& =\mid a\left(t_{2}\right)-a\left(t_{1}\right) \\
& +\int_{0}^{t_{1}} \frac{\left(\phi_{1}\left(t_{2}\right)-\phi_{1}(s)\right)^{\alpha-1}}{\Gamma(\alpha)} f_{1}\left(s, x\left(\psi_{1}(s)\right)\right) \phi_{1}^{\prime}(s) d s \\
& \text { - } \int_{0}^{t_{2}} \frac{\left(\phi_{2}\left(t_{2}\right)-\phi_{2}(s)\right)^{\beta-1}}{\Gamma(\beta)} f_{2}\left(s, x\left(\psi_{2}(s)\right)\right) \phi_{2}^{\prime}(s) d s \\
& +\int_{t_{1}}^{t_{2}} \frac{\left(\phi_{1}\left(t_{2}\right)-\phi_{1}(s)\right)^{\alpha-1}}{\Gamma(\alpha)} f_{1}\left(s, x\left(\psi_{1}(s)\right)\right) \phi_{1}^{\prime}(s) d s \\
& \text { - } \int_{0}^{t_{2}} \frac{\left(\phi_{2}\left(t_{2}\right)-\phi_{2}(s)\right)^{\beta-1}}{\Gamma(\beta)} f_{2}\left(s, x\left(\psi_{2}(s)\right)\right) \phi_{2}^{\prime}(s) d s \\
& -\int_{0}^{t_{1}} \frac{\left(\phi_{1}\left(t_{1}\right)-\phi_{1}(s)\right)^{\alpha-1}}{\Gamma(\alpha)} f_{1}\left(s, x\left(\psi_{1}(s)\right)\right) \phi_{1}^{\prime}(s) d s \\
& \text { - } \quad \int_{0}^{t_{1}} \frac{\left(\phi_{2}\left(t_{1}\right)-\phi_{2}(s)\right)^{\beta-1}}{\Gamma(\beta)} f_{2}\left(s, x\left(\psi_{2}(s)\right)\right) \phi_{2}^{\prime}(s) d s \mid \\
& \leq \mid a\left(t_{2}\right)-a\left(t_{1}\right) \\
& +\int_{0}^{t_{1}} \frac{\left(\phi_{1}\left(t_{1}\right)-\phi_{1}(s)\right)^{\alpha-1}}{\Gamma(\alpha)} f_{1}\left(s, x\left(\psi_{1}(s)\right)\right) \phi_{1}^{\prime}(s) d s \\
& \text { - } \int_{0}^{t_{1}} \frac{\left(\phi_{2}\left(t_{1}\right)-\phi_{2}(s)\right)^{\beta-1}}{\Gamma(\beta)} f_{2}\left(s, x\left(\psi_{2}(s)\right)\right) \phi_{2}^{\prime}(s) d s \\
& +\int_{0}^{t_{1}} \frac{\left(\phi_{1}\left(t_{1}\right)-\phi_{1}(s)\right)^{\alpha-1}}{\Gamma(\alpha)} f_{1}\left(s, x\left(\psi_{1}(s)\right)\right) \phi_{1}^{\prime}(s) d s \\
& \text { - } \quad \int_{t_{1}}^{t_{2}} \frac{\left(\phi_{2}\left(t_{1}\right)-\phi_{2}(s)\right)^{\beta-1}}{\Gamma(\beta)} f_{2}\left(s, x\left(\psi_{2}(s)\right)\right) \phi_{2}^{\prime}(s) d s \\
& +\int_{t_{1}}^{t_{2}} \frac{\left(\phi_{1}\left(t_{2}\right)-\phi_{1}(s)\right)^{\alpha-1}}{\Gamma(\alpha)} f_{1}\left(s, x\left(\psi_{1}(s)\right)\right) \phi_{1}^{\prime}(s) d s \\
& \text { - } \quad \int_{0}^{t_{2}} \frac{\left(\phi_{2}\left(t_{1}\right)-\phi_{2}(s)\right)^{\beta-1}}{\Gamma(\beta)} f_{2}\left(s, x\left(\psi_{2}(s)\right)\right) \phi_{2}^{\prime}(s) d s \\
& -\int_{0}^{t_{1}} \frac{\left(\phi_{1}\left(t_{1}\right)-\phi_{1}(s)\right)^{\alpha-1}}{\Gamma(\alpha)} f_{1}\left(s, x\left(\psi_{1}(s)\right)\right) \phi_{1}^{\prime}(s) d s \\
& \text { - } \quad \int_{0}^{t_{1}} \frac{\left(\phi_{2}\left(t_{1}\right)-\phi_{2}(s)\right)^{\beta-1}}{\Gamma(\beta)} f_{2}\left(s, x\left(\psi_{2}(s)\right)\right) \phi_{2}^{\prime}(s) \mid \\
& \leq\left|a\left(t_{2}\right)-a\left(t_{1}\right)\right| \\
& +\int_{0}^{t_{1}} \frac{\left(\phi_{1}\left(t_{1}\right)-\phi_{1}(s)\right)^{\alpha-1}}{\Gamma(\alpha)}\left|f_{1}\left(s, x\left(\psi_{1}(s)\right)\right)\right| \phi_{1}^{\prime}(s) d s \\
& \text { - } \quad \int_{t_{1}}^{t_{2}} \frac{\left(\phi_{2}\left(t_{1}\right)-\phi_{2}(s)\right)^{\beta-1}}{\Gamma(\beta)}\left|f_{2}\left(s, x\left(\psi_{2}(s)\right)\right)\right| \phi_{2}^{\prime}(s) d s
\end{aligned}
$$




$$
\begin{array}{ll}
+ & \int_{t_{1}}^{t_{2}} \frac{\left(\phi_{1}\left(t_{2}\right)-\phi_{1}(s)\right)^{\alpha-1}}{\Gamma(\alpha)}\left|f_{1}\left(s, x\left(\psi_{1}(s)\right)\right)\right| \phi_{1}^{\prime}(s) d s \\
& \cdot \int_{0}^{t_{2}} \frac{\left(\phi_{2}\left(t_{1}\right)-\phi_{2}(s)\right)^{\beta-1}}{\Gamma(\beta)}\left|f_{2}\left(s, x\left(\psi_{2}(s)\right)\right)\right| \phi_{2}^{\prime}(s) d s \\
\leq & \left|a\left(t_{2}\right)-a\left(t_{1}\right)\right| \\
+ & \int_{0}^{t_{1}} \frac{\left(\phi_{1}\left(t_{1}\right)-\phi_{1}(s)\right)^{\alpha-1}}{\Gamma(\alpha)}\left|f_{1}\left(s, x\left(\psi_{1}(s)\right)\right)\right| \phi_{1}^{\prime}(s) d s \\
& \cdot \int_{t_{1}}^{t_{2}} \frac{\left(\phi_{2}\left(t_{1}\right)-\phi_{2}(s)\right)^{\beta-1}}{\Gamma(\beta)}\left|f_{2}\left(s, x\left(\psi_{2}(s)\right)\right)\right| \phi_{2}^{\prime}(s) d s \\
+ & \int_{t_{1}}^{t_{2}} \frac{\left(\phi_{1}\left(t_{1}\right)-\phi_{1}(s)\right)^{\alpha-1}}{\Gamma(\alpha)}\left|f_{1}\left(s, x\left(\psi_{1}(s)\right)\right)\right| \phi_{1}^{\prime}(s) d s \\
\cdot & \int_{0}^{t_{2}} \frac{\left(\phi_{2}\left(t_{1}\right)-\phi_{2}(s)\right)^{\beta-1}}{\Gamma(\beta)}\left|f_{2}\left(s, x\left(\psi_{2}(s)\right)\right)\right| \phi_{2}^{\prime}(s) d s \\
+ & \int_{t_{1}}^{t_{2}} \frac{\left(\phi_{1}\left(t_{1}\right)-\phi_{1}(s)\right)^{\alpha-1}}{\Gamma(\alpha)}\left|f_{1}\left(s, x\left(\psi_{1}(s)\right)\right)\right| \phi_{1}^{\prime}(s) d s \\
& \int_{t_{1}}^{t_{2}} \frac{\left(\phi_{2}\left(t_{1}\right)-\phi_{2}(s)\right)^{\beta-1}}{\Gamma(\beta)}\left|f_{2}\left(s, x\left(\psi_{2}(s)\right)\right)\right| \phi_{2}^{\prime}(s) d s .
\end{array}
$$

Then we obtain

$$
\begin{aligned}
\left|(\mathbb{T} x)\left(t_{2}\right)-(\mathbb{T} x)\left(t_{1}\right)\right| \leq & \left|a\left(t_{2}\right)-a\left(t_{1}\right)\right| \\
+ & {\left[\frac{M_{1} T^{\alpha-\gamma_{1}}}{\Gamma\left(\alpha-\gamma_{1}+1\right)}+\frac{b_{1} r T^{\alpha}}{\Gamma(\alpha+1)}\right] } \\
& {\left[\frac{M_{2}\left(\phi_{2}\left(t_{1}\right)-\phi_{2}\left(t_{2}\right)\right)^{\beta-\gamma_{2}}}{\Gamma\left(\beta-\gamma_{2}+1\right)}+\frac{b_{2} r\left(\phi_{2}\left(t_{1}\right)-\phi_{2}\left(t_{2}\right)\right)^{\beta}}{\Gamma(\beta+1)}\right] } \\
+ & {\left[\frac{M_{1}\left(\phi_{1}\left(t_{1}\right)-\phi_{1}\left(t_{2}\right)\right)^{\alpha-\gamma_{1}}}{\Gamma\left(\alpha-\gamma_{1}+1\right)}+\frac{b_{1} r\left(\phi_{1}\left(t_{1}\right)-\phi_{1}\left(t_{2}\right)\right)^{\alpha}}{\Gamma(\alpha+1)}\right] } \\
\cdot & {\left[\frac{M_{2}\left(\phi_{2}\left(t_{1}\right)-\phi_{2}\left(t_{2}\right)\right)^{\beta-\gamma_{2}}}{\Gamma\left(\beta-\gamma_{2}+1\right)}+\frac{b_{2} r\left(\phi_{2}\left(t_{1}\right)-\phi_{2}\left(t_{2}\right)\right)^{\beta}}{\Gamma(\beta+1)}\right] } \\
+ & {\left[\frac{M_{1}\left(\phi_{1}\left(t_{1}\right)-\phi_{1}\left(t_{2}\right)\right)^{\alpha-\gamma_{1}}}{\Gamma\left(\alpha-\gamma_{1}+1\right)}+\frac{b_{1} r\left(\phi_{1}\left(t_{1}\right)-\phi_{1}\left(t_{2}\right)\right)^{\alpha}}{\Gamma(\alpha+1)}\right] } \\
& \cdot\left[\frac{M_{2}\left(\phi_{2}\left(t_{1}\right)-\phi_{2}\left(t_{2}\right)\right)^{\beta-\gamma_{2}}}{\Gamma\left(\beta-\gamma_{2}+1\right)}+\frac{b_{2} r\left(\phi_{2}\left(t_{1}\right)-\phi_{2}\left(t_{2}\right)\right)^{\beta}}{\Gamma(\beta+1)}\right] .
\end{aligned}
$$

Then

$$
\left|(\mathbb{T} x)\left(t_{2}\right)-(\mathbb{T} x)\left(t_{1}\right)\right| \rightarrow 0 \quad \text { as } \quad t_{2} \rightarrow t_{1} .
$$

This means that the functions from $\mathbb{T} S_{r}$ are equi-continuous on $J$. Then, by the ArzelaAscoli Theorem [14], the closure of $\mathbb{T} S_{r}$ is compact.

It is clear that the set $S_{r}$ is nonempty, bounded, closed and convex.

Assumptions (ii) and (iv) imply that $\mathbb{T}: S_{r} \rightarrow C(J)$ is a continuous operator in $x$.

Since all conditions of the Schauder fixed-point theorem hold, then $\mathbb{T}$ has a fixed point in $S_{r}$.

\section{Special Cases and Remarks}

In Section 2, we prove an existence result for the functional quadratic $\phi$ - integral equation of fractional order (2) which in turn gives the existence as well as the existence of many key integral and functional equations that arise in nonlinear analysis and its applications. 
Corollary 1. Let the assumptions ( $i$-(vii) be satisfied with $\psi_{1}=\psi_{2}=\psi$ then there exists at least one solution for the functional quadratic $\phi$ - integral equation of fractional order

$$
\begin{aligned}
x(t)= & a(t)+\int_{0}^{t} \frac{\left(\phi_{1}(t)-\phi_{1}(s)\right)^{\alpha-1}}{\Gamma(\alpha)} f_{1}(s, x(\psi(s))) \phi_{1}^{\prime}(s) d s \\
& \cdot \int_{0}^{t} \frac{\left(\phi_{2}(t)-\phi_{2}(s)\right)^{\beta-1}}{\Gamma(\beta)} f_{2}(s, x(\psi(s))) \phi_{2}^{\prime}(s) d s, t \in J .
\end{aligned}
$$

Corollary 2. Let the assumptions ( $i$ )-(vii) be satisfied with $\psi_{1}=\psi_{2}=\psi, \phi_{1}=\phi_{2}=\phi$ and

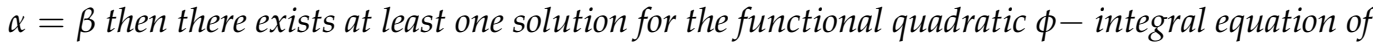
fractional order

$$
x(t)=a(t)+\left(\int_{0}^{t} \frac{\left(\phi_{1}(t)-\phi_{1}(s)\right)^{\alpha-1}}{\Gamma(\alpha)} f_{1}(s, x(\psi(s))) \phi_{1}^{\prime}(s) d s\right)^{2} t \in J .
$$

Corollary 3. Let the assumptions (i)-(vii) be satisfied with $\phi_{1}(t)=\phi_{2}(t)=\phi(t), \psi_{1}(t)=$ $\psi_{2}(t)=t$ then there exists at least one solution for the functional quadratic $\phi-$ integral equation of fractional order

$$
\begin{aligned}
x(t)= & a(t)+\int_{0}^{t} \frac{(\phi(t)-\phi(s))^{\alpha-1}}{\Gamma(\alpha)} f_{1}(s, x(s)) \phi^{\prime}(s) d s \\
& \int_{0}^{t} \frac{(\phi(t)-\phi(s))^{\beta-1}}{\Gamma(\beta)} f_{2}(s, x(s)) \phi^{\prime}(s) d s, t \in J .
\end{aligned}
$$

Corollary 4. Let the assumptions (i)-(vii) be satisfied with $\phi_{1}(t)=\phi_{2}(t)=t^{m}, m>0$ then there exists at least one solution for the Erdélyi-Kober functional quadratic equation of fractional order

$$
\begin{aligned}
x(t)= & a(t)+\int_{0}^{t} \frac{\left(t^{m}-s^{m}\right)^{\alpha-1}}{\Gamma(\alpha)} f_{1}\left(s, x\left(\psi_{1}(s)\right)\right) m s^{m-1} d s \\
& \cdot \quad \int_{0}^{t} \frac{\left(t^{m}-s^{m}\right)^{\beta-1}}{\Gamma(\beta)} f_{2}\left(s, x\left(\psi_{2}(s)\right)\right) m s^{m-1} d s, t \in J .
\end{aligned}
$$

Corollary 5. Let the assumptions (i)-(vii) be satisfied with $\phi_{1}(t)=\phi_{2}(t)=t^{m}, m>0$ and $\psi_{1}(t)=\psi_{2}(t)=t$ then there exists at least one solution for the Erdélyi-Kober functional quadratic equation of fractional order

$$
\begin{aligned}
x(t)= & a(t)+\int_{0}^{t} \frac{\left(t^{m}-s^{m}\right)^{\alpha-1}}{\Gamma(\alpha)} f_{1}(s, x(s)) m s^{m-1} d s \\
& \int_{0}^{t} \frac{\left(t^{m}-s^{m}\right)^{\beta-1}}{\Gamma(\beta)} f_{2}(s, x(s)) m s^{m-1} d s, t \in J .
\end{aligned}
$$

Corollary 6. Let the assumptions (i)-(vii) be satisfied with $\phi_{1}(t)=\phi_{2}(t)=t, \psi_{1}=\psi_{2}=\psi$ then there exists at least one solution for the functional quadratic integral equation of fractional order

$$
x(t)=a(t)+\int_{0}^{t} \frac{(t-s)^{\alpha-1}}{\Gamma(\alpha)} f_{1}(s, x(\psi(s))) d s . \int_{0}^{t} \frac{(t-s)^{\beta-1}}{\Gamma(\beta)} f_{2}(s, x(\psi(s))) d s, t \in J .
$$

The same result is obtained in [15].

Corollary 7. Let the assumptions (i)-(vii) be satisfied with $\phi_{1}(t)=\phi_{2}(t)=t, \psi_{1}(t)=\psi_{2}(t)=t$ then there exists at least one solution for the functional quadratic integral equation of fractional order

$$
x(t)=a(t)+\int_{0}^{t} \frac{(t-s)^{\alpha-1}}{\Gamma(\alpha)} f_{1}(s, x(s)) d s . \int_{0}^{t} \frac{(t-s)^{\beta-1}}{\Gamma(\beta)} f_{2}(s, x(s)) d s, t \in J .
$$


The same result is obtained in [7].

Letting $\alpha, \beta \rightarrow 1$, we obtain

Corollary 8. Let the assumptions (i)-(vii) be satisfied with $\alpha, \beta \rightarrow 1$, and $\psi_{1}(t)=\psi_{2}(t)=t$ and letting $\alpha, \beta \rightarrow 1$, then there exists at least one solution for the functional quadratic integral equation of fractional order

$$
x(t)=a(t)+\int_{0}^{t} f_{1}(s, x(s)) d s . \int_{0}^{t} f_{2}(s, x(s)) d s, t \in J .
$$

The same result is obtained in [13]. When $a(t)=0, f_{1}=f_{2}$, we have

$$
\sqrt{x(t)}=\int_{0}^{t} f_{1}(s, x(s)) d s, t \in J
$$

\section{Properties of Solutions}

In this section, we give the sufficient conditions for the uniqueness of the solution of the quadratic integral Equation (2) and study some of its properties.

\subsection{Uniqueness of Solutions of QFIE (2)}

Let us assume the following assumptions

(i*) $\quad a: J \rightarrow R_{+}$is continuous and $\sup _{t \in J}|a(t)|=k$;

(ii*) $f_{1}, f_{2}: J \times R \rightarrow R_{+}$satisfy the Carathéodory condition (i.e., measurable in $t$ for all $x \in R$ and continuous in $x$ for all $t \in J)$.

(iii*) There exist two nonnegative constants $\lambda_{1}, \lambda_{2} \in L^{1}$ such that

$$
\left|f_{i}(t, x)-f_{i}(t, y)\right| \leq \lambda_{i}|x-y|, \forall x, y \in R, t \in J
$$

(iv $\left.{ }^{*}\right) \phi_{i}: J \rightarrow J, i=1,2$ are increasing and absolutely continuous.

$\left(\mathrm{v}^{*}\right) \quad \psi_{i}: J \rightarrow J, i=1,2$ are continuous.

$\left(\mathrm{vi}^{*}\right) m_{i}(t)=\left|f_{i}(t, 0)\right|, \forall t \in J, I_{\phi_{i}}^{\gamma_{i}} m_{i} \leq M_{i}, i=1,2 \forall \gamma_{1} \leq \alpha, \gamma_{2} \leq \beta$.

$\left(\right.$ vii $\left.^{*}\right) r$ is a positive solution of the inequality:

$$
\begin{aligned}
k & +\frac{M_{1} M_{2} T^{\alpha+\beta-\gamma_{1}-\gamma_{2}}}{\Gamma\left(\alpha-\gamma_{1}+1\right) \Gamma\left(\beta-\gamma_{2}+1\right)}+\frac{b_{1} M_{2} r T^{\alpha+\beta-\gamma_{2}}}{\Gamma(\alpha+1) \Gamma\left(\beta-\gamma_{2}+1\right)} \\
& +\frac{b_{2} M_{1} r T^{\beta+\alpha-\gamma_{1}}}{\Gamma(\beta+1) \Gamma\left(\alpha-\gamma_{1}+1\right)}+\frac{b_{1} b_{2} r^{2} T^{\alpha+\beta}}{\Gamma(\alpha+1) \Gamma(\beta+1)} \leq r .
\end{aligned}
$$

Theorem 3. Let the assumptions (i)-(vii) be satisfied. If

$$
\begin{gathered}
\frac{\lambda_{2} \lambda_{1} r T^{\beta+\alpha}}{\Gamma(\beta+1) \Gamma(\alpha+1)}+\frac{M_{1} \lambda_{2} T^{\alpha+\beta-\gamma_{1}}}{\Gamma\left(\alpha-\gamma_{1}+1\right) \Gamma(\beta+1)} \\
+\frac{\lambda_{2} \lambda_{1} r T^{\beta+\alpha}}{\Gamma(\beta+1) \Gamma(\alpha+1)}+\frac{M_{2} \lambda_{1} T^{\alpha+\beta-\gamma_{2}}}{\Gamma\left(\beta-\gamma_{2}+1\right) \Gamma(\alpha+1)}<1,
\end{gathered}
$$

then the quadratic integral Equation (2) has a unique positive solution $x \in C(J)$.

Proof.

$$
\begin{aligned}
\left|f_{i}(t, x)-f_{i}(t, 0)\right| & \leq \lambda_{i}|x-0| \\
\left|f_{i}(t, x)\right| & \leq\left|f_{i}(t, 0)\right|+\lambda_{i}|x| \\
& \leq m_{i}(t)+\lambda_{i}|x|, m_{i}(t)=\left|f_{i}(t, 0)\right|, \forall x \in R, t \in J .
\end{aligned}
$$


Equation (2) can be written as

$$
\begin{aligned}
x(t) & =a(t)+I_{\phi_{1}}^{\alpha} f_{1}\left(t, x\left(\psi_{1}(t)\right)\right) I_{\phi_{2}}^{\beta} f_{2}\left(t, x\left(\psi_{2}(t)\right)\right) \\
& =a(t)+I_{\phi_{1}}^{\alpha-\gamma_{1}} I_{\phi_{1}}^{\gamma_{1}} f_{1}\left(t, x\left(\psi_{1}(t)\right)\right) I_{\phi_{1}}^{\beta-\gamma_{2}} I_{\phi_{2}}^{\gamma_{2}} f_{2}\left(t, x\left(\psi_{2}(t)\right)\right), t \in J .
\end{aligned}
$$

Define the operator $F$ by:

$$
\begin{aligned}
F x(t)= & a(t)+\int_{0}^{t} \frac{\left(\phi_{1}(t)-\phi_{1}(s)\right)^{\alpha-1}}{\Gamma(\alpha)} f_{1}\left(s, x\left(\psi_{1}(s)\right)\right) \phi_{1}^{\prime}(s) d s \\
& \cdot \int_{0}^{t} \frac{\left(\phi_{2}(t)-\phi_{2}(s)\right)^{\beta-1}}{\Gamma(\beta)} f_{2}\left(s, x\left(\psi_{2}(s)\right)\right) \phi_{2}^{\prime}(s) d s, t \in J .
\end{aligned}
$$

The operator $F$ maps $C(J)$ into itself. For this, let $t_{1}, t_{2} \in J, t_{1}<t_{2}$ such that $\left|t_{2}-t_{1}\right| \leq$ $\delta$, then in similar way as before using the condition (vi) and the relation (3), we can prove that

$$
\left|(F x)\left(t_{2}\right)-(F x)\left(t_{1}\right)\right| \rightarrow 0 \text { as } t_{2} \rightarrow t_{1} .
$$

Which proves that the operator $F$ is continuous.

Now, we will prove that $F$ is a contraction. Let $x, y \in C(J)$, then we have

$$
\begin{aligned}
|F x(t)-F y(t)|= & \mid \int_{0}^{t} \frac{\left(\phi_{1}(t)-\phi_{1}(s)\right)^{\alpha-1}}{\Gamma(\alpha)} f_{1}\left(s, x\left(\psi_{1}(s)\right)\right) \phi_{1}^{\prime}(s) d s \\
& \cdot \int_{0}^{t} \frac{\left(\phi_{2}(t)-\phi_{2}(s)\right)^{\beta-1}}{\Gamma(\beta)} f_{2}\left(s, x\left(\psi_{2}(s)\right)\right) \phi_{2}^{\prime}(s) d s \\
& -\int_{0}^{t} \frac{\left(\phi_{1}(t)-\phi_{1}(s)\right)^{\alpha-1}}{\Gamma(\alpha)} f_{1}\left(s, y\left(\psi_{1}(s)\right)\right) \phi_{1}^{\prime}(s) d s \\
& \cdot \int_{0}^{t} \frac{\left(\phi_{2}(t)-\phi_{2}(s)\right)^{\beta-1}}{\Gamma(\beta)} f_{2}\left(s, y\left(\psi_{2}(s)\right)\right) \phi_{2}^{\prime}(s) d s \\
& +\int_{0}^{t} \frac{\left(\phi_{1}(t)-\phi_{1}(s)\right)^{\alpha-1}}{\Gamma(\alpha)} f_{1}\left(s, x\left(\psi_{1}(s)\right)\right) \phi_{1}^{\prime}(s) d s \\
& \cdot \int_{0}^{t} \frac{\left(\phi_{2}(t)-\phi_{2}(s)\right)^{\beta-1}}{\Gamma(\beta)} f_{2}\left(s, y\left(\psi_{2}(s)\right)\right) \phi_{2}^{\prime}(s) d s \\
- & \int_{0}^{t} \frac{\left(\phi_{1}(t)-\phi_{1}(s)\right)^{\alpha-1}}{\Gamma(\alpha)} f_{1}\left(s, x\left(\psi_{1}(s)\right)\right) \phi_{1}^{\prime}(s) d s \\
& \cdot \int_{0}^{t} \frac{\left(\phi_{2}(t)-\phi_{2}(s)\right)^{\beta-1}}{\Gamma(\beta)} f_{2}\left(s, y\left(\psi_{2}(s)\right)\right) \phi_{2}^{\prime}(s) d s \mid \\
\leq & \int_{0}^{t} \frac{\left(\phi_{1}(t)-\phi_{1}(s)\right)^{\alpha-1}}{\Gamma(\alpha)}\left|f_{1}\left(s, x\left(\psi_{1}(s)\right)\right)\right| \phi_{1}^{\prime}(s) d s \\
& \cdot \int_{0}^{t} \frac{\left(\phi_{2}(t)-\phi_{2}(s)\right)^{\beta-1}}{\Gamma(\beta)}\left|f_{2}\left(s, x\left(\psi_{2}(s)\right)\right)-f_{2}\left(s, y\left(\psi_{2}(s)\right)\right)\right| \phi_{2}^{\prime}(s) d s
\end{aligned}
$$




$$
\begin{aligned}
& +\int_{0}^{t} \frac{\left(\phi_{1}(t)-\phi_{1}(s)\right)^{\alpha-1}}{\Gamma(\alpha)}\left|f_{1}\left(s, x\left(\psi_{1}(s)\right)\right)-f_{1}\left(s, y\left(\psi_{1}(s)\right)\right)\right| \phi_{1}^{\prime}(s) d s \\
& \text { - } \int_{0}^{t} \frac{\left(\phi_{2}(t)-\phi_{2}(s)\right)^{\beta-1}}{\Gamma(\beta)}\left|f_{2}\left(s, y\left(\psi_{2}(s)\right)\right)\right| \phi_{2}^{\prime}(s) d s \\
& \leq \lambda_{2} \int_{0}^{t} \frac{\left(\phi_{1}(t)-\phi_{1}(s)\right)^{\alpha-1}}{\Gamma(\alpha)}\left|f_{1}\left(s, x\left(\psi_{1}(s)\right)\right)\right| \phi_{1}^{\prime}(s) d s \\
& \text { - } \int_{0}^{t} \frac{\left(\phi_{2}(t)-\phi_{2}(s)\right)^{\beta-1}}{\Gamma(\beta)}\left|x\left(\psi_{2}(s)\right)-y\left(\psi_{2}(s)\right)\right| \phi_{2}^{\prime}(s) d s \\
& +\quad \lambda_{1} \int_{0}^{t} \frac{\left(\phi_{1}(t)-\phi_{1}(s)\right)^{\alpha-1}}{\Gamma(\alpha)}\left|x\left(\psi_{1}(s)\right)-y\left(\psi_{1}(s)\right)\right| \phi_{1}^{\prime}(s) d s \\
& \text { - } \quad \int_{0}^{t} \frac{\left(\phi_{2}(t)-\phi_{2}(s)\right)^{\beta-1}}{\Gamma(\beta)}\left|f_{2}\left(s, y\left(\psi_{2}(s)\right)\right)\right| \phi_{2}^{\prime}(s) d s \\
& \leq \lambda_{2}|| x-y \| \int_{0}^{t} \frac{\left(\phi_{1}(t)-\phi_{1}(s)\right)^{\alpha-1}}{\Gamma(\alpha)}\left|f_{1}\left(s, x\left(\psi_{1}(s)\right)\right)\right| \phi_{1}^{\prime}(s) d s \\
& \int_{0}^{t} \frac{\left(\phi_{2}(t)-\phi_{2}(s)\right)^{\beta-1}}{\Gamma(\beta)} \phi_{2}^{\prime}(s) d s \\
& +\quad \lambda_{1}\|x-y\| \int_{0}^{t} \frac{\left(\phi_{1}(t)-\phi_{1}(s)\right)^{\alpha-1}}{\Gamma(\alpha)} \phi_{1}^{\prime}(s) d s \\
& \text { - } \int_{0}^{t} \frac{\left(\phi_{2}(t)-\phi_{2}(s)\right)^{\beta-1}}{\Gamma(\beta)}\left|f_{2}\left(s, y\left(\psi_{2}(s)\right)\right)\right| \phi_{2}^{\prime}(s) d s \\
& \leq \frac{\lambda_{2}|| x-y||}{\Gamma(\beta+1)} \int_{0}^{t} \frac{\left(\phi_{1}(t)-\phi_{1}(s)\right)^{\alpha-1}}{\Gamma(\alpha)}\left|f_{1}\left(s, x\left(\psi_{1}(s)\right)\right)\right| \phi_{1}^{\prime}(s) d s \\
& +\frac{\lambda_{1}|| x-y||}{\Gamma(\alpha+1)} \int_{0}^{t} \frac{\left(\phi_{2}(t)-\phi_{2}(s)\right)^{\beta-1}}{\Gamma(\beta)}\left|f_{2}\left(s, y\left(\psi_{2}(s)\right)\right)\right| \phi_{2}^{\prime}(s) d s \\
& \leq \frac{\lambda_{2} T^{\beta}\|x-y\|}{\Gamma(\beta+1)}\left[\frac{T^{\alpha} \lambda_{1} r}{\Gamma(\alpha+1)}+\frac{M_{1} T^{\alpha-\gamma_{1}}}{\Gamma\left(\alpha-\gamma_{1}+1\right)}\right] \\
& +\frac{\lambda_{1} T^{\alpha}\|x-y\|}{\Gamma(\alpha+1)}\left[\frac{M_{2} T^{\beta-\gamma_{2}}}{\Gamma\left(\beta-\gamma_{2}+1\right)}+\frac{\lambda_{2} T^{\beta} r}{\Gamma(\beta+1)}\right] \\
& \leq\left[\frac{\lambda_{2} \lambda_{1} r T^{\beta+\alpha}}{\Gamma(\beta+1) \Gamma(\alpha+1)}+\frac{M_{1} \lambda_{2} T^{\alpha+\beta-\gamma_{1}}}{\Gamma\left(\alpha-\gamma_{1}+1\right) \Gamma(\beta+1)}\right. \\
& \left.+\frac{\lambda_{2} \lambda_{1} r T^{\beta+\alpha}}{\Gamma(\beta+1) \Gamma(\alpha+1)}+\frac{M_{2} \lambda_{1} T^{\alpha+\beta-\gamma_{2}}}{\Gamma\left(\beta-\gamma_{2}+1\right) \Gamma(\alpha+1)}\right]\|x-y\| \text {. }
\end{aligned}
$$

Then

$$
|F x(t)-F y(t)| \leq \Lambda\|x-y\|, \quad \Lambda \in(0,1)
$$

where

$$
\begin{aligned}
\Lambda & =\frac{\lambda_{2} \lambda_{1} r T^{\beta+\alpha}}{\Gamma(\beta+1) \Gamma(\alpha+1)}+\frac{M_{1} \lambda_{2} T^{\alpha+\beta-\gamma_{1}}}{\Gamma\left(\alpha-\gamma_{1}+1\right) \Gamma(\beta+1)} \\
& +\frac{\lambda_{2} \lambda_{1} r T^{\beta+\alpha}}{\Gamma(\beta+1) \Gamma(\alpha+1)}+\frac{M_{2} \lambda_{1} T^{\alpha+\beta-\gamma_{2}}}{\Gamma\left(\beta-\gamma_{2}+1\right) \Gamma(\alpha+1)}
\end{aligned}
$$

Then $F$ is a contraction. Therefore, by the Banach contraction fixed point Theorem [8], the operator $F$ has a unique fixed point $x \in C(J)$ (i.e., the quadratic integral Equation (2) has a unique solution $x \in C(J))$. which completes the proof. 
4.2. Maximal and Minimal Solutions

Definition 2 ([16]). Let $q(t)$ be a solution $x(t)$ of $(2)$ Then $q(t)$ is said to be a maximal solution of (2) if every solution of (2) on J satisfies the inequality $x(t) \leq q(t), t \in J$. A minimal solution $s(t)$ can be defined in a similar way by reversing the above inequality, i.e., $x(t) \geq s(t), t \in J$.

We need the following lemma to prove the existence of maximal and minimal solutions of (2).

Lemma 1. Let $f_{i}(t, x), i=1,2$ satisfy the assumptions in Theorem 2 and let $x(t), y(t)$ be continuous functions on $J$ satisfying

$$
\begin{aligned}
& x(t) \leq a(t)+I_{\phi_{1}}^{\alpha} f_{1}\left(t, x\left(\psi_{1}(t)\right)\right) I_{\phi_{2}}^{\beta} f_{2}\left(t, x\left(\psi_{2}(t)\right)\right) \\
& y(t) \geq a(t)+I_{\phi_{1}}^{\alpha} f_{1}\left(t, y\left(\psi_{1}(t)\right)\right) I_{\phi_{2}}^{\beta} f_{2}\left(t, y\left(\psi_{2}(t)\right)\right)
\end{aligned}
$$

where one of them is strict.

Suppose $f_{i}(t, x)$ is a nondecreasing function in $x$. Then

$$
x(t)<y(t), t \in J .
$$

Proof. Let the conclusion (4) be false; then there exists $t_{1}$ such that

$$
x\left(t_{1}\right)=y\left(t_{1}\right), \quad t_{1}>0
$$

and

$$
x(t)<y(t), \quad 0<t<t_{1} .
$$

From the monotonicity of the function $f_{i}$ in $x$, we obtain

$$
\begin{aligned}
x\left(t_{1}\right) & \leq a\left(t_{1}\right)+I_{\phi_{1}}^{\alpha} f_{1}\left(t_{1}, x\left(\psi_{1}\left(t_{1}\right)\right)\right) I_{\phi_{2}}^{\beta} f_{2}\left(t_{1}, x\left(\psi_{2}\left(t_{1}\right)\right)\right) \\
& <a\left(t_{1}\right)+I_{\phi_{1}}^{\alpha} f_{1}\left(t_{1}, x\left(\psi_{1}\left(t_{1}\right)\right)\right) I_{\phi_{2}}^{\beta} f_{2}\left(t_{1}, x\left(\psi_{2}\left(t_{1}\right)\right)\right) \\
& <y\left(t_{1}\right) .
\end{aligned}
$$

This contradicts the fact that $x\left(t_{1}\right)=y\left(t_{1}\right)$; then

$$
x(t)<y(t), t \in J .
$$

Theorem 4. Let the assumptions of Theorem 1 be satisfied. Furthermore, if $f_{i}, i=1,2$ is $a$ nondecreasing function in $x$, then there exist maximal and minimal solutions of (2).

Proof. Firstly, we shall prove the existence of the maximal solution of (2). Let $\epsilon>0$ be true. Now consider the fractional-order quadratic functional integral equation

$$
\begin{aligned}
x_{\epsilon}(t)= & a(t)+\int_{0}^{t} \frac{\left(\phi_{1}(t)-\phi_{1}(s)\right)^{\alpha-1}}{\Gamma(\alpha)} f_{1_{\epsilon}}\left(s, x_{\epsilon}\left(\psi_{1}(s)\right)\right) \phi_{1}^{\prime}(s) d s \\
& \int_{0}^{t} \frac{\left(\phi_{2}(t)-\phi_{2}(s)\right)^{\beta-1}}{\Gamma(\beta)} f_{2_{\epsilon}}\left(s, x_{\epsilon}\left(\psi_{2}(s)\right)\right) \phi_{2}^{\prime}(s) d s, t \in J,
\end{aligned}
$$

where

$$
f_{i_{\epsilon}}\left(t, x_{\epsilon}\left(\psi_{i}(t)\right)\right)=f_{i}\left(t, x_{\epsilon}\left(\psi_{i}(t)\right)\right)+\epsilon, i=1,2 .
$$

Clearly the functions $f_{i_{\epsilon}}\left(t, x_{\epsilon}\right), i=1,2$ satisfy assumptions (ii), (iv) and

$$
\left|f_{i_{\epsilon}}\left(t, x_{\epsilon}\right)\right| \leq m_{i}(t)+\epsilon+b_{i}|x|=m_{i}^{\prime}(t)+b|x| .
$$


Therefore, the quadratic integral Equation (5) has a continuous solution $x_{\epsilon}(t)$ according to Theorem 2.

Let $\epsilon_{1}$ and $\epsilon_{2}$ be such that $0<\epsilon_{2}<\epsilon_{1}<\epsilon$. Then

$$
\begin{gathered}
x_{\epsilon_{1}}(t)=a(t)+I_{\phi_{1}}^{\alpha} f_{1_{\epsilon_{1}}}\left(t, x_{\epsilon_{1}}\left(\psi_{1}(t)\right)\right) I_{\phi_{2}}^{\beta} f_{2_{\epsilon_{1}}}\left(t, x_{\epsilon_{1}}\left(\psi_{2}(t)\right)\right), \\
x_{\epsilon_{1}}(t)=a(t)+I_{\phi_{1}}^{\alpha} f_{1_{\epsilon_{1}}}\left(t, x_{\epsilon_{1}}\left(\psi_{1}(t)\right)\right) I_{\phi_{2}}^{\beta} f_{2_{\epsilon_{1}}}\left(t, x_{\epsilon_{1}}\left(\psi_{2}(t)\right)\right), \\
=a(t)+I_{\phi_{1}}^{\alpha}\left(f_{1}\left(t, x_{\epsilon_{1}}\left(\psi_{1}(t)\right)+\epsilon_{1}\right) I_{\phi_{2}}^{\beta}\left(f_{2}\left(t, x_{\epsilon_{1}}\left(\psi_{2}(t)\right)\right)+\epsilon_{1}\right),\right. \\
>a(t)+I_{\phi_{1}}^{\alpha}\left(f_{1}\left(t, x_{\epsilon_{1}}\left(\psi_{1}(t)\right)+\epsilon_{2}\right) I_{\phi_{2}}^{\beta}\left(f_{2}\left(t, x_{\epsilon_{1}}\left(\psi_{2}(t)\right)\right)+\epsilon_{2}\right),\right. \\
x_{\epsilon_{2}}(t)=a(t)+I_{\phi_{1}}^{\alpha}\left(f_{1}\left(t, x_{\epsilon_{2}}\left(\psi_{1}(t)\right)\right)+\epsilon_{2}\right) I_{\phi_{2}}^{\beta}\left(f_{2}\left(t, x_{\epsilon_{2}}\left(\psi_{2}(t)\right)\right)+\epsilon_{2}\right) .
\end{gathered}
$$

Applying Lemma 1, (6) and (7) then we obtain

$$
x_{\epsilon_{2}}(t)<x_{\epsilon_{1}}(t) \quad \text { for } t \in J
$$

As shown before in the proof of Theorem 1, the family of functions $x_{\epsilon}(t)$ defined by (5) is uniformly bounded and of equi-continuous functions. Hence by the Arzela-Ascoli Theorem, there exists a decreasing sequence $\epsilon_{n}$ such that $\epsilon_{n} \rightarrow 0$ as $n \rightarrow \infty$, and $\lim _{n \rightarrow \infty} x_{\epsilon_{n}}(t)$ exists uniformly in $J$. We denote this limit by $q(t)$. From the continuity of the functions $f_{1, \epsilon_{n}}$ and $f_{2, \epsilon_{n}}$ in the second argument, we obtain

$$
q(t)=\lim _{n \rightarrow \infty} x_{\epsilon_{n}}(t)=a(t)+I_{\phi_{1}}^{\alpha} f_{1}\left(t, q\left(\psi_{1}(t)\right)\right) I_{\phi_{2}}^{\beta} f_{2}\left(t, q\left(\psi_{2}(t)\right)\right)
$$

which proves that $q(t)$ is a solution of (2).

Finally, we shall show that $q(t)$ is maximal solution of (2). To do this, let $x(t)$ be any solution of (1). Then

$$
\begin{aligned}
x_{\epsilon}(t) & =a(t)+I_{\phi_{1}}^{\alpha} f_{1_{\epsilon}}\left(t, x_{\epsilon}\left(\psi_{1}(t)\right)\right) I_{\phi_{2}}^{\beta} f_{2_{\epsilon}}\left(t, x_{\epsilon}\left(\psi_{2}(t)\right)\right) \\
& >a(t)+I_{\phi_{1}}^{\alpha} f_{1}\left(t, x_{\epsilon}\left(\psi_{1}(t)\right)\right) I_{\phi_{2}}^{\beta} f_{2}\left(t, x_{\epsilon}\left(\psi_{2}(t)\right)\right) \\
x(t) & =a(t)+I_{\phi_{1}}^{\alpha} f_{1}\left(t, x\left(\psi_{1}(t)\right)\right) I_{\phi_{2}}^{\beta} f_{2}\left(t, x\left(\psi_{2}(t)\right)\right)
\end{aligned}
$$

Applying Lemma 1, we obtain

$$
x_{\epsilon}(t)>x(t) \quad \text { for } t \in J .
$$

From the uniqueness of the maximal solution (see $[16,17])$, it is clear that $x_{\epsilon}(t)$ tends to $q(t)$ uniformly in $t \in J$ as $\epsilon \rightarrow 0$.

In a similar way we can prove that there exists a minimal solution of (2).

\section{Conclusions}

Fractional integral differential equations have been studied in many studies and monographs (see [18-21]). Especially quadratic integral equations of fractional order, for example $[7,10,15,18,20]$.

In this work, we discussed a $\phi$ - fractional order quadratic integral equation. Some exiting results were established by constructing an iterative scheme in aim of proving the analogous result for the Carathéodory theorem [14], and by applying Banach contraction mapping to demonstrate the existence of the unique solution of that equation. Furthermore, the existence of maximal and minimal solutions of the $\phi-$ fractional order quadratic integral equation is proved. 
Author Contributions: Author Contributions: Conceptualization, A.M.A.E.-S., H.H.G.H. and S.M.A.I. All authors have read and agreed to the published version of the manuscript.

Funding: This research received no external funding.

Acknowledgments: The authors thank the editors and the reviewers for their useful comments that improved our work.

Conflicts of Interest: The authors declare no conflict of interest.

\section{References}

1. Argyros, I.K. Quadratic equations and applications to Chandrasekhar's and related equations. Bull. Austral. Math. Soc. 1985, 32, 275-292. [CrossRef]

2. Argyros, I.K. On a class of quadratic integral equations with perturbations. Funct. Approx. 1992, 20, 51-63.

3. Banas, J.; Lecko, M.; El-Sayed, W.G. Existence theorems of some quadratic integral equation. J. Math. Anal. Appl. 1998, 227, 276-279. [CrossRef]

4. Banaś, J.; Martinon, A. Monotonic Solutions of a quadratic Integral Equation of Volterra Type. Comput. Math. Appl. 2004, 47, 271-279. [CrossRef]

5. Banaś, J.; Caballero, J.; Rocha, J.; Sadarangani, K. Monotonic Solutions of a Class of Quadratic Integral Equations of Volterra Type. Comput. Math. Appl. 2005, 49, 943-952. [CrossRef]

6. Chandrasekhar, S. Radiative Transfer; Oxford University Press: London, UK, 1950.

7. El-Sayed, A.M.A.; Mohamed, M.S.; Mohamed, F.F.S. Existence of positive continuous solution of a quadratic integral equation of fractional orders. J. Fract. Calc. Appl. 2011, 1, 1-7.

8. Goebel, K.; Kirk, W.A. Topics in Metric Fixed Point Theory; Cambridge University Press: Cambridge, UK, 1990.

9. Hashem, H.H.G. On the solution of a generalized fractional order integral equation and some applications. J. Fract. Calc. Appl. 2015, 6, 120-130.

10. Hashem, H.H.G.; El-Sayed, A.M.A. Existence results for a quadratic integral equation of fractional order by a certain function Fixed Point Theory 2020, 21, 181-190. [CrossRef]

11. Hashem, H.H.G.; Zaki, M.S. Carathéodory theorem for quadratic integral equations of ErdyéliKober type. J. Fract. Calc. Appl. 2013, 4, 1-8.

12. Caballero, J.; Mingarelli, A.B.; Sadarangani, K. Existence of solutions of an integral equation of Chandrasekhar type in the theory of radiative transfer. Electr. J. Differ. Equ. 2006, 57, 1-11.

13. El-Sayed, A.M.A.; Hashem, H.H.G. Carathèodory type theorem for a nonlinear quadratic integral equation. Math. Sci. Res. J. 2008, 12, 71-95.

14. Curtain, R.F.; Pritchard, A.J. Functional Analysis in Modern Applied Mathematics; Academic Press: London, UK; Cambridge, MA, USA, 1977.

15. El-Sayed, A.M.A.; Hashem, H.H.G.; Omar, Y.M.Y. Positive continuous solution of a quadratic integral equation of fractional orders. Math. Sci. Lett. 2013, 2, 1-9. [CrossRef]

16. Lakshmikantham, V.; Leela, S. Differential and Integral Inequalities; Academic Press: New York, NY, USA; London, UK, 1969; Volume 1.

17. Rao, M.R. Ordinary Differential Equations; East-West Press Pvt Ltd., New Delhi-Madras: New Delhi, India, 1980.

18. Banaś, J.; Rzepka, B. Monotonic solutions of a quadratic integral equations of fractional order. J. Math. Anal. Appl. 2007, 332, 1370-1378. [CrossRef]

19. El-Sayed, A.M.A.; Gaafar, F.M.; Hashem, H.H.G. On the maximal and minimal solutions of arbitrary-orders nonlinear functional integral and differenbtial equations. Math. Sci. Res. J. 2004, 8, 336-348.

20. El-Sayed, A.M.A.; Hashem, H.H.G. Existence results for nonlinear quadratic functional integral equations of fractional order. Miskolc Math. Notes 2013, 14, 79-87. [CrossRef]

21. El-Sayed, A.M.A.; Hashem, H.H.G.; Al-Issa, S.M. An Implicit Hybrid Delay Functional Integral Equation: Existence of Integrable Solutions and Continuous Dependence. Mathematics 2021, 9, 3234. [CrossRef] 